\title{
Large volume 3D characterization by plasma FIB DualBeam microscopy
}

\author{
T L Burnett ${ }^{1,2}$, R Kelley ${ }^{3}$, B Winiarski ${ }^{1,2}$, M Daly ${ }^{1}, \mathrm{~K} \mathrm{Mani}^{3}$, P J Withers ${ }^{1}$ \\ 1. The University of Manchester, Materials Department, Manchester, M13 9PL, UK \\ 2. FEI Company, Achtseweg Noord 5, Bldg 5651 GG, Eindhoven, The Netherlands \\ 3. FEI Company, 5350 Northeast Dawson Creek Drive, Hillsboro, OR 97124, USA
}

There is a critical need to analyze many material systems in three dimensions (3D), for example to understand connectivity of phases, porous networks and complex shapes. There are several tools available for 3D characterization e.g. X-ray computed Tomography (CT) [1], Serial Sectioning SEM Tomography (SST) [2, 3], transmission electron microscopy [4] and Atom Probe [5]. Figure 1 demonstrates their complementarity in terms of the lengthscales they can access.

The emergence of dual beam Focused ion beam (FIB-SEM) using Gallium ions has provided a means of accessing regions of interest of $50 \mu \mathrm{m}$ dimensions both for site specific TEM and SST in the SEM with slice thicknesses down to $10 \mathrm{~nm}$. Although destructive it has enables 3D imaging of structure, grain structure (via EBSD) and chemistry (by EDS). However the limited volumes accessible through the Gallium Ion FIB present a severe limitation. The size of the volume that can be examined via SST and the depth from which the regions of interest identified by X-ray CT is also key to opening up possibilities through a correlative tomography framework [1].

There are many scenarios in materials science that require analysis across length-scales up to many 100's of microns that lie beyond what a Ga ion FIB can typically access, relating to grain microtextures, grain neighborhoods, grain boundaries, inclusions and cracks.

In this paper we present the first examination of the capabilities of a new type of FIB-SEM that utilizes a Xe Plasma beam FIB (PFIB) for machining alongside an electron imaging column. We show that the FEI Helios PFIB can achieve a throughput 20-50x that of a Ga ion FIB opening up regions 100s of microns beneath the surface and allowing serial section tomography over volumes of hundreds of micron dimensions. The performance of the PFIB is demonstrated through the study of an austenitic and a ferritic steel both used within the energy sector.

The PFIB was operated at $30 \mathrm{kV}$ and $59 \mathrm{nA}$ beam current to collect typically 100's of slices $\sim 100 \times$ $100 \mu^{2}$ cross section size and a slice thickness of $80 \mathrm{~nm}$. In contrast to typical SST within a FIB a stage rocking mode $\left(+/-5^{\circ}\right)$ is sued to minimize curtaining that may be created during unidirectional FIB milling. Images using the Through lens detector (TLD) were captures at high resolution with the pixel size $<40 \mathrm{~nm}$. These SEM-PFIB settings allowed us to collect 10-20 slices per hour (Fig. 2).

The study here focuses on a ferritic steel used in pressure vessels with the region analyzed e just below a ductile fracture surface (Fig. 3). The distribution of carbides, as well as the voids associated with the ductile fracture, were imaged and analyzed. Our results indicate that curtain-free surfaces with a high detail level of microstructural features, e.g. carbides at the sized at $100 \mathrm{~nm}$. Results shows that the PFIB SST technique produces low levels of surface damage as evidenced by good channeling contrast. We will also present results showing Kikuchi patterns from the machined surface.

Other ongoing experimental studies across a wide range of materials from paint to ceramics suggest the PFIB can routinely and quickly provide 3D serial section tomographs over dimensions of many hundreds of microns helping bridge the gap between conventional FIB and X-ray tomography. All results were aligned and analyzed using FEI-Avizo Visualization software. 
1. E. Maire and P. J. Withers: 'Quantitative X-ray tomography', Int. Mater. Rev., 2014, 59, 1, 1-43.

2. M. D. Uchic, L. Holzer, B. J. Inkson, E. L. Principe, P. Munroe 'Three-Dimensional Microstructural Characterization Using Focused Ion Beam Tomography’ MRS Bulletin, Volume 32, Issue 05, May 2007, pp 408-416

3. Borgh, I., et al., On the three-dimensional structure of WC grains in cemented carbides. Acta Mat., 2013. 61: 4726-4733.

4. C. Kübel, A. Voigt, R. Schoenmakers, M. Otten, D. Su, T-C. Lee, A. Carlsson, J. Bradley 'Recent Advances in Electron Tomography: TEM and HAADF-STEM Tomography for Materials Science and Semiconductor Applications' Microscopy and Microanalysis, 11, Issue 05, 2005, pp 378-400

5. Blavettea, D., Duguay, S., Atom probe tomography in nanoelectronics. European Physical Journal - Applied Physics, 2014. 68(1): p. 10101-p1-12.

6. Burnett, T.L., McDonald, S.A., Gholinia, A., Geurts, R., Janus, M., Slater, T., Haigh, S.J., Ornek, C., Almuaili, F., Engelberg, D.L., Thompson, G.E., Withers, P.J., Correlative Tomography. Scientific Reports, 2014. 4: p. 4711

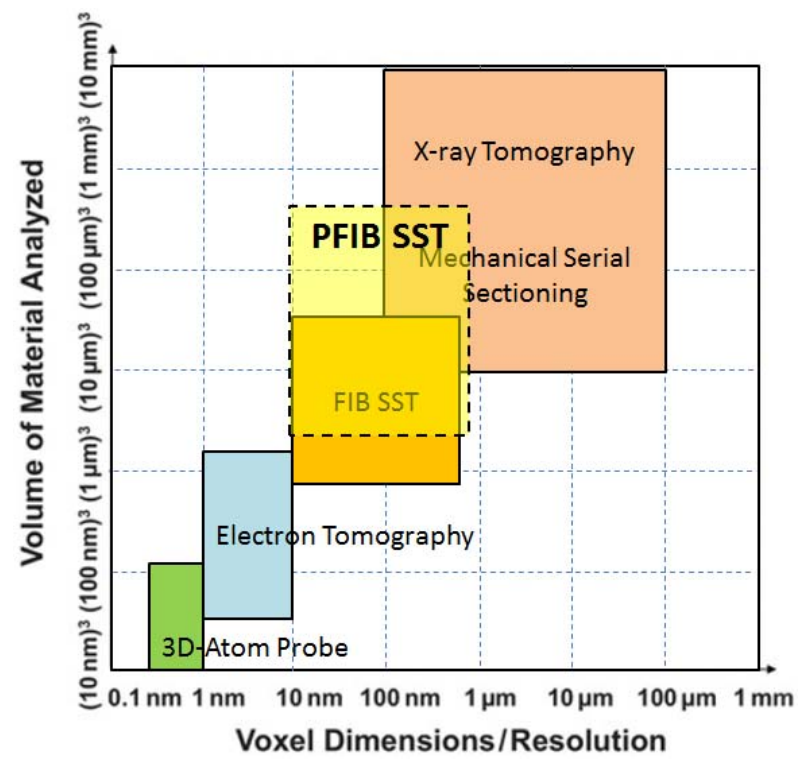

Figure 1. Comparison of Plasma focused ion beam (PFIB) tomography with other 3D microscopy methods.

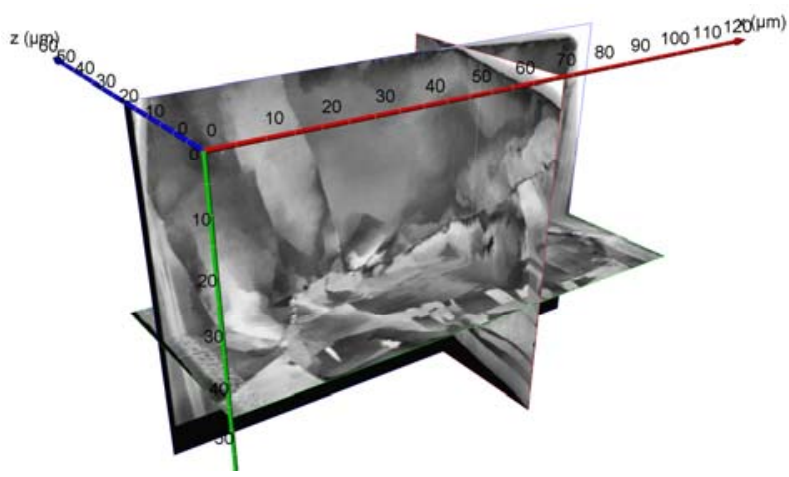

Figure 2. Avizo visualisation of 440 slices of an austenitic stainless steel sectioned with FEI Helios plasma FIB DualBeam at 59 nA Plasma current and $30 \mathrm{kV}$ electric field. The slice thickness is $100 \mathrm{~nm}$ and the sections face is $100 \times 60{\mu \mathrm{m}^{2}}^{2}$ with the pixel size of $38 \mathrm{~nm}$. Combined PFIB milling and SEM imaging of a single slice is about 6 minutes. FEGSEM imaging at $2 k V, 0.68 n A, E T D$ detector, field-free mode. The inset shows a Kikuich pattern from the point.
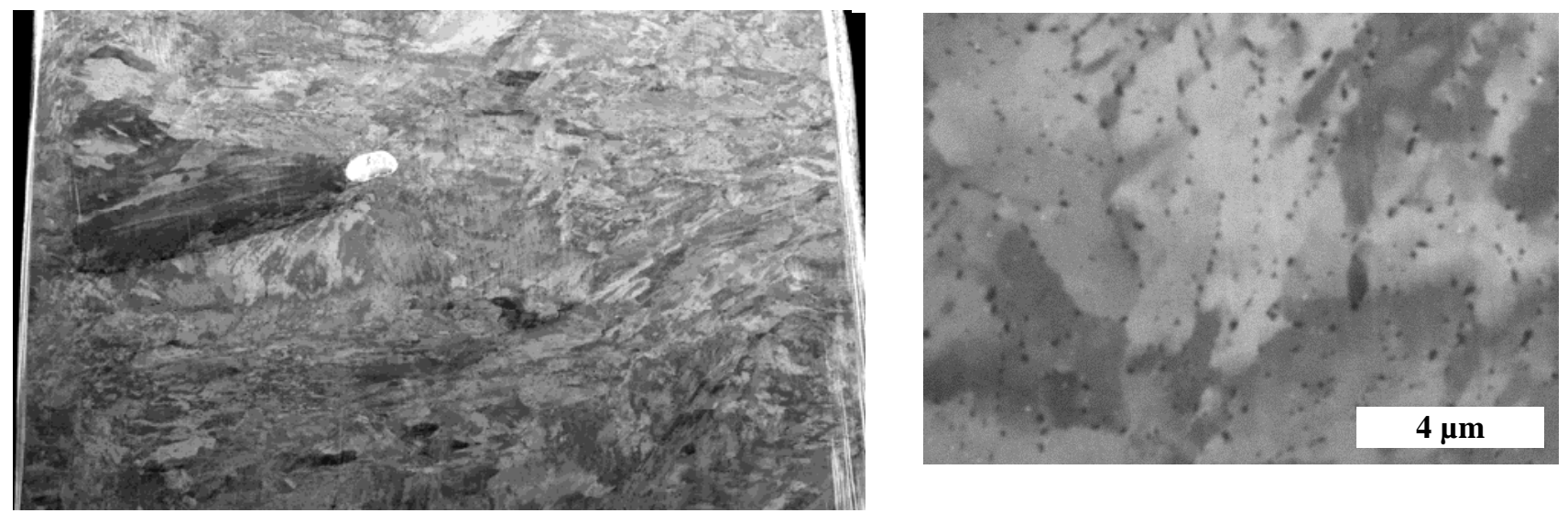

Figure 3. Ferritic steel from a nuclear pressure vessel sectioned with FEI Helios plasma FIB DualBeam at 59 nA Plasma current and $30 \mathrm{kV}$. The slice thickness is $100 \mathrm{~nm}$ and the sections face is $100 \times 96 \mu \mathrm{m}^{2}$ with the pixel size of $29 \mathrm{~nm}$. Combined PFIB milling and SEM imaging of a single slice is about 8 minutes. FEGSEM imaging at $2 \mathrm{kV}$, $0.80 \mathrm{nA}, T L D$ detector and field-free mode. 